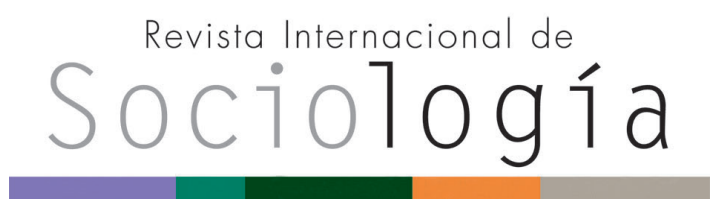

Revista Internacional de Sociología RIS

vol. 78 (2), e157, abril-junio, 2020, ISSN-L:0034-9712

https://doi.org/10.3989/ris.2020.78.2.18.164

\section{EL MECANISMO DE INVERSIÓN IDEOLÓGICA: SOBRE LOS PROCESOS DE LEGITIMACIÓN DEL CAPITALISMO}

\author{
ISMAEL PUGA \\ Universidad Central de Chile \\ ismael.puga@ucentral.cl \\ ORCID iD: https://orcid.org/0000-0002-6385-9678

\section{CRISTÓBAL MOYA} \\ Zeppelin Universität \\ cristobal.moya@zu.de \\ ORCID iD: https://orcid.org/0000-0002-7176-4775
}

\section{THE IDEOLOGICAL INVESTMENT MECHANISM: ABOUT THE LEGITIMATION PROCESSES OF CAPITALISM}

Cómo citar este artículo / Citation: Puga, I. y C. Moya. 2020. "El mecanismo de inversión ideológica: sobre los procesos de legitimación del capitalismo". Revista Internacional de Sociología 78(2):e157. https://doi. org/10.3989/ris.2020.78.2.18.164

\section{RESUMEN}

El estudio de la legitimación del capitalismo como explicación de su estabilidad ha sido desplazado por estudios sobre las disposiciones individuales hacia la desigualdad y las jerarquías. Recuperando el carácter explicativo de la legitimación, proponemos distinguir analíticamente entre tres grandes mecanismos de legitimación del orden social: legitimidad social, disimulación e inversión ideológica. Estos mecanismos comparten principios clave para la teoría sociológica clásica, pero difieren en la explicación sobre cómo el capitalismo obtiene validez social y se sostiene en el tiempo. Proponemos específicamente la primacía explicativa del mecanismo de inversión ideológica como tesis más plausible para la estabilidad social en las sociedades contemporáneas. A partir de esta tesis, sugerimos una nueva agenda de investigación teórica y empírica sobre la legitimación del orden capitalista.

\section{Palabras Clave}

Cambio social; Ideología; Legitimación; Mecanismos; Reproducción.
Copyright: @ 2020 CSIC. Este es un artículo de acceso abierto distribuido bajo los términos de la licencia de uso y distribución Creative Commons Reconocimiento 4.0 Internacional (CC BY 4.0).

Recibido: 26/10/2018. Aceptado: 20/10/2019 Publicado online: 22/06/2020

\section{Abstract}

The study of legitimation as a mechanism for explaining the stability of capitalism has been largely displaced by studies on individuals' dispositions towards inequality. Returning to the explanative role of legitimation, we propose an analyti-cal distinction between three mechanisms of legitimation of the social order: social legitimacy, dissimulation, and ideo-logical inversion. These mechanisms share some key fea-tures inspired by classical sociology, while differing in their explanation on how capitalism obtains social validity and endures through time. Specifically, we propose the primacy of the ideological inversion mechanism, as the most plausi-ble thesis for the stability of contemporary societies. From this viewpoint, we suggest a new agenda for theoretical and empirical research on the legitimation of capitalism.

\section{KEYWORDS}

Ideology; Legitimacy; Legitimation; Mechanisms; Reproduction. 


\section{INTRODUCCIÓN}

Este artículo realiza una crítica de las interpretaciones contemporáneas del problema de la legitimación social del orden y sus aplicaciones en la investigación empírica. Se plantea que el estudio de la legitimación social, en cuanto mecanismo explicativo de la estabilización del capitalismo, ha sido desplazado por un estudio de las disposiciones individuales respecto del orden social, lo que deja un vacío en la teoría sociológica.

Desde una perspectiva de sociología analítica, proponemos una integración teórica que distingue entre tres grandes mecanismos de legitimación en la teoría sociológica, posibilitando su contraste y comparación explicativa en estudios empíricos.

El tercer mecanismo presentado, la inversión ideológica, corresponde a una nueva interpretación analítica de la idea de inversión ideológica en Marx. Este mecanismo permite la reproducción del orden sin que exista realmente un consenso social sobre su justificación: es un mecanismo de legitimación sin legitimidad social. Proponemos como tesis la primacía explicativa de este tercer mecanismo, mayormente omitido por la tradición sociológica y hasta ahora escasamente especificado, y desarrollamos las implicaciones teóricas y las hipótesis empíricas que se derivan de esta tesis central.

\section{EL PROBLema de LA LEgITIMACIÓN EN LA SOCIOLOGÍA}

En gran medida, la teoría social moderna nació con la pregunta por el sostenimiento del orden. Frente al declive de las justificaciones divinas y tradicionales del antiguo régimen, surgen preguntas como: ¿qué sostiene a las sociedades modernas? y ¿cómo se estabilizan y reproducen, pese a los conflictos que contienen? Este proceso, mediante el cual las sociedades se sostienen en el tiempo con el consentimiento general de sus miembros, se ha denominado habitualmente 'legitimación'.

La tradición sociológica ha desarrollado distintas respuestas al problema de la legitimación del orden social, que podemos trazar hasta los clásicos de la disciplina. Una de las primeras es la de Weber $(1918,1920)$, según la cual las jerarquías sociales buscan activamente producir creencias en su legitimidad para asegurar su propia reproducción, y que continúa siendo particularmente incidente en la teoría social contemporánea y especialmente en la investigación empírica sobre los procesos de legitimación (Johnson, Dowd y Ridgeway 2006). El supuesto generalmente compartido es que, para mantener su estabilidad, un sistema de poder requiere lograr ciertos niveles de legitimidad social.

La legitimidad social implica que el sistema puede ser justificado en los términos de las creencias de quienes lo conforman, llevando a una validación del orden. Se requiere entonces un cierto consenso social en torno a la pertinencia y justificación del orden. Además, según Beetham (2013), la legitimidad se produce a través de otras dos dimensiones. Una es la dimensión práctica: los individuos muestran consentimiento hacia el orden a través de prácticas concretas como votar en elecciones y, en general, actuando de manera afín a este orden. Otra es la dimensión legal, que contribuye a la legitimación del orden social en tanto este se ajuste a unas reglas establecidas.

Tomando distancia de este supuesto común, nos interesa especialmente cómo Marx (1844, 1859, 1867) abordó el problema de la reproducción del orden, debido a las consecuencias que tiene para el debate contemporáneo sobre la legitimación del orden social.

La cuestión de la reproducción del capitalismo y sus tensiones atraviesa la totalidad de la obra de Marx. Sin embargo, no encontraremos un escrito sistemático sobre los procesos que aseguran la connivencia del proletariado ante la sociedad burguesa: no hay un texto rector en que Marx defina el problema de la ideología y desarrolle acabadamente su relación con la reproducción de las relaciones de producción capitalistas. Esto ha significado, por una parte, que la relevancia del problema ideológico no está consensuada en la teoría marxista. Por otra parte, ha llevado a interpretaciones marxistas de la reproducción ideológica del orden sumamente disímiles (Mouffe 1979; Gramsci 1971; Althusser 1970; Lukács 1923).

\section{LA BRECHA EN LOS ESTUDIOS DEL CONFLICTO SOCIAL Y LA LEGITIMACIÓN DEL ORDEN}

A riesgo de simplificar, distinguimos tres momentos del estudio de la legitimación del orden social. Primero, clásicos como Weber y Marx abordaron el problema desde una perspectiva histórica, buscando comprender la emergencia y reproducción del capitalismo. En el segundo, autores como Foucault (1975), Bourdieu (1979; 1980) o Homans (1958), entre otros, complementaron esta perspectiva con dinámicas microsociales y estudios empíricos de la producción de distintas creencias y actitudes sobre las estructuras sociales. Emergen en un tercer momento dos tendencias divergentes. Por un lado, desde la tradición marxista (Burawoy 1979; 2012) y desde la sociología pragmática francesa (Boltanski 2011; Boltanski y Chiapello, 1999; Boltanski y Thévenot, 1999) surgen actualizaciones críticas de las perspectivas antes mencionadas. Estas destacan por el énfasis en los aspectos subjetivos de la legitimación de la dominación social. Por el otro, surge un productivo campo de estudios sobre las evaluaciones de la justicia, considerando sus antecedentes y consecuencias. Los llamados estudios de justicia social empírica (Müller 
y Wegener 1995; Wegener 1987; Jasso 1978; 2008; 2015) se concentran en la justicia distributiva; así como lo hace una psicología de la legitimidad, expandiendo su foco analítico a dimensiones procedimentales e interaccionales de la justicia (Almstrom 2006; Bénabou y Tirole 2006; Jost, Banaji y Nosek 2004; Sidanius y Pratto 2001; Lerner 1980). En la actualidad, estos dos grandes enfoques empíricos dominan la investigación aplicada sobre legitimación de la desigualdad en Europa y los EE. UU. (Arnett y Sidanius, 2018; Schneider y Valet, 2017; Osborne y Sibley, 2015; Cichocka y Jost, 2014).

Podemos observar aquí un desplazamiento en el objeto de estudio desde el cambio y la estabilidad social hacia el desarrollo de actitudes o disposiciones individuales. Weber (1922) introdujo el concepto de legitimidad social para explicar la estabilidad de estructuras y reglas que implicaban subordinación. En cambio, enfatizando ya sea el estudio psicométrico de las evaluaciones de justicia o las motivaciones psicológicas para aceptar el statu quo, los estudios de la justicia social empírica y de la psicología de la legitimidad abordan disposiciones estrictamente individuales. Se ha tendido así a traducir la idea de legitimación como la producción de actitudes legitimantes. No obstante, Weber (1920) es explícito en que las motivaciones individuales no son suficientemente estables para sostener un orden: se requiere reconocer el orden como socialmente válido -i.e., como aceptado por el colectivo-. Todos los clásicos, en su propio lenguaje teórico, reconocen el carácter social de la legitimación, aún difiriendo en cómo se produce.

Ciertamente, el estudio de las disposiciones individuales ante las desigualdades y estructuras es un campo fructífero y relevante de por sí. Lo problemático es que rara vez se evalúa directamente la relevancia de estas disposiciones para explicar la emergencia concreta de conflictos sociales. En un exhaustivo metaanálisis de la evidencia sobre predictores psicosociales de la acción colectiva, Van Zomeren, Postmes y Spears (2008) reportan que muy pocos de los más de 180 estudios revisados predicen acciones concretas. Tampoco estos estudios utilizan como variables explicativas las ideas o preferencias internalizadas sobre estructuras y desigualdades sociales; en cambio, tienden a abordar grupos específicos prediciendo disposiciones a participar en acciones colectivas, con base en atributos de percepción de justicia, identidad colectiva o percepción de efectividad. Solo el primer atributo se asocia a la legitimación, entendida como la capacidad del orden de aparecer justificado ante los actores ${ }^{1}$. La relevancia del desarrollo de las identidades colectivas es indudable, pero responde a otro factor, mientras que la efectividad percibida agrupa indiferenciadamente un conjunto de posibles explicaciones de la conflictividad (entre las que está la percepción de validez social del orden).
Finalmente, entre los estudios que buscan explicar efectivamente las conductas, Van Zomeren, Postmes y Spears observan que: (a) en general, los efectos estudiados no se sostienen tras controles básicos; (b) incluso sin controles, los efectos son considerablemente menores que en aquellos estudios que predicen disposiciones hacia el orden, y que (c) la relevancia de las percepciones de justicia es particularmente débil. Así, como mínimo existe una amplia brecha de factores no observados entre la capacidad de entender la disposición a movilizarse por cambios y la movilización efectiva. Como los clásicos afirmaran, los procesos de movilización social no parecen producirse sencillamente por la agregación de individuos disconformes.

El problema en estas perspectivas, sin embargo, es más profundo. La literatura psicosocial en boga renuncia, finalmente, a una explicación sistemática de la legitimación del orden cuando omite el problema de cómo las mismas estructuras sociales producen, entre los actores, las disposiciones individuales que estudia. En este sentido, la precaución analítica de Weber respecto al estudio de la legitimación continúa vigente: si buscamos entender el proceso que asegura la reproducción de un orden, limitarnos a las motivaciones individuales de los actores solo puede llevarnos a respuestas parciales y contingentes. Un mecanismo explicativo adecuado necesariamente ha de gatillarse por las mismas estructuras sociales que poseen la capacidad de permanecer y reproducirse. Existen en la tradición sociológica un número importante de teorías que abordan el problema de la reproducción del orden desde esta precaución. Si bien es imposible una revisión exhaustiva de las mismas aquí, en la siguiente sección propondremos la distinción de dos grandes mecanismos sociales de legitimación entre ellas.

\section{APROXIMACIÓN ANALÍTICA: LOS MECA- NISMOS SOCIALES DE LEGITIMACIÓN}

Como mencionamos, los clásicos de la sociología coinciden en la relevancia del problema de la validez social. Es decir, en que la conducta conformista de un actor ante un orden dado no se basa únicamente en sus preferencias individuales, sino también en el reconocimiento de que dicho orden posee validez colectiva, con base presumiblemente en preferencias comunes.

Así, por ejemplo, es posible concebir a un trabajador que no protesta contra su situación de explotación laboral porque ha incorporado valores $y$ creencias que justifican las relaciones capitalistas de trabajo. En este caso, las creencias del trabajador producen su consentimiento respecto al orden, pues lo hacen aceptable y soportable, o incluso deseable. Sin embargo, esta conducta también puede ser producto de una situación en que no existe propiamente 
el consentimiento del trabajador, pero este sí cree que otros validan dicho orden social y lo respaldarán, ya sea por acción u omisión.

A este mecanismo general, al efecto de la validez social sobre la reproducción del orden, le designaremos legitimación. Distintos mecanismos sociales de legitimación explicarán cómo se reproduce regularmente la validez del orden social, particularmente en el capitalismo moderno.

De esta aproximación general no se deriva que las disposiciones individuales sean irrelevantes en los procesos de reproducción y cambio social. Sin embargo, en la medida en que una relación o institución social goza de validez, las disposiciones internas tendrán menos relevancia a la hora de explicar la conducta individual; incluso quienes posean disposiciones individuales a activar el conflicto tenderán a la inmovilidad (Berger y Zelditch, 1998). Cuando la validez social se resquebraja, dichas disposiciones individuales cobran mayor relevancia.

Como el estudio de la legitimación busca comprender la reproducción y el cambio social, los mecanismos de legitimación no pueden ser sencillamente asociaciones entre determinadas disposiciones y conductas (individuales o colectivas). Estos mecanismos especifican cómo y por qué se reproduce de manera estable un orden social, y cómo se inhibe el potencial conflictivo de sus jerarquías internas. Por lo tanto, establecen un vínculo explicativo que comienza en la misma existencia de un orden dado —con jerarquías y estructuras sociales establecidas-, y culmina en un consenso práctico. Por consenso práctico entendemos la producción regular y generalizada de prácticas que actualizan el orden social, a la vez tendiendo a inhibir las acciones orientadas al cambio. Por un lado, este consenso práctico reproduce materialmente el orden social, por ejemplo, sosteniendo relaciones jerárquicas en la producción y marcando las distancias sociales en las interacciones sociales. Por otro lado, comunica efectivamente la validez social del orden al mostrarle a los individuos que los otros obedecen a través de sus prácticas.

En este sentido, cuando Beetham (2013) discute el concepto de legitimidad social, está en lo correcto al relevar el aspecto práctico del proceso, pero su propuesta es imprecisa al identificar este aspecto como una dimensión que opera en paralelo a otra dimensión del consenso social (entendido como un consenso de las creencias). Dicha imprecisión impide distinguir la legitimidad social propiamente tal de otras explicaciones del proceso de legitimación que Beetham no está contemplando. EI consenso práctico corresponde al último paso de un mecanismo de legitimación, y este último paso puede producirse como resultado de un consenso social, así como puede producirse a través de otros mecanismos. En términos analíticos, el consenso social no debe entenderse en paralelo al consenso práctico, sino que corresponde a una tesis explicativa sobre este último. El consenso práctico, por otra parte, resulta suficiente para la reproducción del orden independientemente de si es producido a través de un consenso social.

El mecanismo general de legitimación, entonces, se basa en la idea de validez social producida a nivel colectivo, y puede representarse esquemáticamente como en la figura 1. Los distintos mecanismos sociales de legitimación corresponden a diferentes explicaciones específicas del funcionamiento de la caja negra que permite a un orden social producir un consenso práctico que, a su vez, actualiza el orden social tendiendo a reproducirlo.

Podemos ilustrar este esquema utilizando el ejemplo del propietario de una mina y sus trabajadores. El consenso práctico representa la obediencia concreta de los empleados a las reglas que el propietario, apoyado en un marco legal, ha definido sobre cuándo llegar a la faena, quién realiza qué trabajos, durante cuánto tiempo, etc. Se expresa

Figura 1

Modelo general (inespecífico) del mecanismo de legitimación

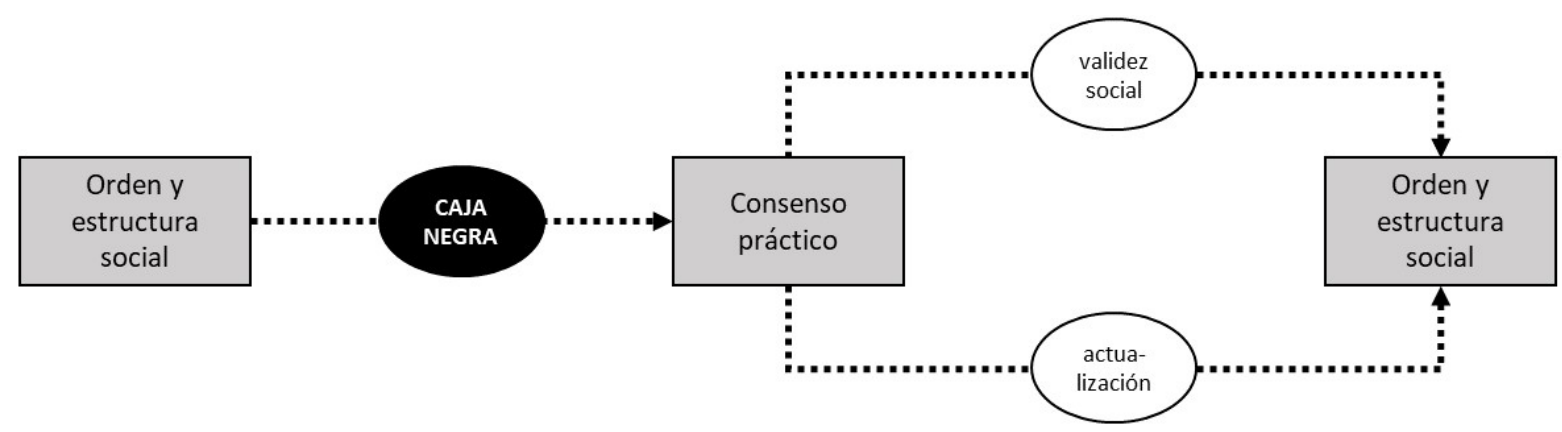

Leyenda

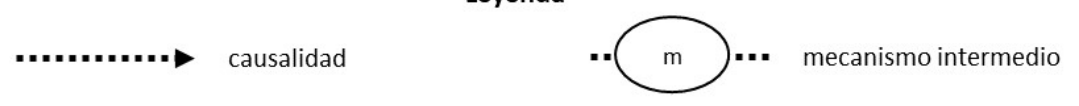

RIS [online] 2020, 78 (2), e157. REVISTA INTERNACIONAL DE SOCIOLOGÍA. ISSN-L: 0034-9712 
también en el hecho de que los trabajadores aceptan el salario ofrecido por este trabajo, y en el trato diferente que tienen con el propietario. Estas prácticas concretas de los trabajadores, por una parte, actualizan el funcionamiento del orden: la mina produce, el propietario se enriquece, los trabajadores obedecen y reciben un salario por ello. Por otra parte, colectivamente estas prácticas demuestran a los trabajadores que el orden es válido, que sus pares parecen aceptarlas. Incluso si un trabajador individual considera que la situación es injusta, se ve inhibido por la evidente aceptación del orden que sus compañeros expresan en la práctica. Si decide individualmente no llegar más a trabajar, la obediencia de los otros asegurará que la mina siga funcionando para beneficio del propietario, mientras que él sufrirá las consecuencias de perder su salario. Cuando nos preguntamos por distintos mecanismos de legitimación, nos preguntamos por qué los trabajadores llegan a obedecer, trabajar y aceptar sus salarios en primer lugar. La caja negra contiene la explicación que conecta la existencia misma de la mina, el propietario y los trabajadores, con la práctica legitimante de estos últimos.

El más intuitivo mecanismo de legitimación que distinguimos en la teoría sociológica es el de la legitimidad social. En este caso, los actores sociales comparten un conjunto de disposiciones normativas y preferencias que justifica adecuadamente el funcionamiento de las estructuras sociales. A dicho conjunto de disposiciones le llamaremos 'consenso normativo'. El consenso normativo no es nunca completo, pero el mecanismo de legitimidad social se caracteriza porque los actores reconocen adecuadamente la validez social del orden y, en consecuencia, se ven compelidos a reproducirlo en su práctica concreta incluso si individualmente lo rechazan. Así, en un capitalismo liberal en que exista efectivamente un consenso normativo sobre la pertinencia y justificación de la democracia representativa, un anarquista que crea firmemente en la democracia directa puede votar en las elecciones o, cuando menos, aceptar en la práctica a las autoridades electas. Esto no lo hace porque crea en la justificación de la democracia representativa, sino porque reconoce la validación social de dicho orden político y la consiguiente futilidad de ignorarlo.

Esta formulación mínima del mecanismo de la legitimidad social puede trazarse desde diversas fuentes. El concepto de consciencia colectiva en Durkheim (1893) refiere precisamente al mencionado consenso normativo. El contenido de dicha consciencia colectiva se deriva, en gran medida, de las transformaciones estructurales de la sociedad y, aunque la vulneración de los principios del consenso normativo es un fenómeno esperable, su frecuencia está limitada por la (potencial) reacción social en contra de esta: mediante el mecanismo de la validez social. La reacción social misma refuerza, a su vez, la vigencia de la validez social. Para Weber (1920), en cambio, los actores en posiciones de poder asegurarán activamente el establecimiento de normas que justifiquen el orden. Establecidas dichas normas, los actores se verán compelidos por la validez social del orden a reproducirlo, cuenten o no con motivaciones individuales para hacerlo. Parsons (1979) intentó sintetizar (algo forzadamente) la visión de Durkheim y Weber, asignando a un sistema específico de mantenimiento de pautas la reproducción del consenso normativo.

Pese a sus diferencias, estas y otras perspectivas sociológicas comparten una noción mínima del proceso de legitimación: (a) el orden social tiende a producir un consenso normativo que lo justifica adecuadamente; (b) los actores tienden a compartir el contenido de dicho consenso normativo y refuerzan cotidianamente la validez social de este en su práctica; (c) la validez social del consenso $-\mathrm{y}$, por tanto, del orden- estabiliza las prácticas y asegura la reproducción social de las estructuras clave. Esta definición mínima del mecanismo de legitimidad social basta para distinguirlo analíticamente de otros mecanismos relevantes, y se representa esquemáticamente en la figura 2.

En la figura 2 observamos que el orden tiende a definir un consenso normativo. El mecanismo específico tras dicha definición varía (como varía entre Weber, Durkheim y Parsons), pero en todo caso hay afinidad $(\approx)$ entre el consenso y el orden: es decir, las normas permiten justificar el funcionamiento del statu quo tal como es. Por esto, y mediante el mecanismo de la validez social (1), dicho consenso normativo produce un consenso práctico adecuado: dado que la validez social respalda estas creencias y normas que justifican el statu quo, los actores tenderán a reproducir este en su práctica, incluso si esto entra en conflicto con sus intereses o disposiciones individuales. Este consenso práctico, a su vez, actualiza concretamente el orden y produce un segundo momento de validez social (2), inhibiendo la acción disruptiva.

Continuando el ejemplo de la mina, en este caso los trabajadores, en general, consideran justo y adecuado que el propietario defina las normas de trabajo y el salario adecuado, ya que han incorporado valores como la disciplina laboral y el respeto por las leyes, dentro del que se encuentra el respeto de la propiedad privada. Existe una relación de afinidad entre la estructura social (la situación concreta de la mina y los trabajadores insertos en una formación social) y el consenso normativo que comparten. Por esto, la mayoría obedece las normas del propietario y del Estado (validez social 1). Con el tiempo, la misma obediencia generalizada hará aún más difícil que un trabajador disconforme intente cambiar las cosas (validez social 2). 
Figura 2.

Mecanismo de legitimidad social

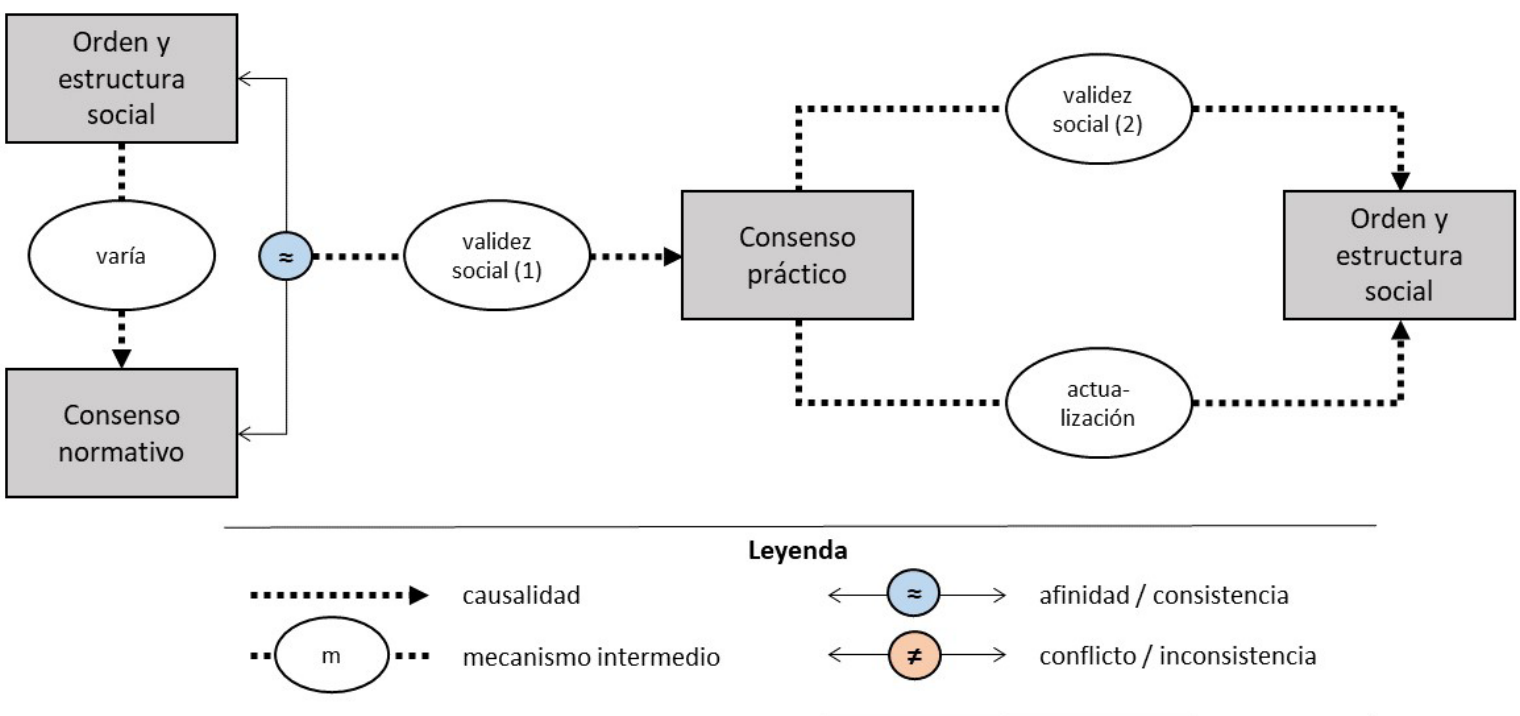

Debemos destacar un supuesto importante del mecanismo de legitimidad social: los actores disciernen aproblemáticamente la correspondencia entre el consenso normativo y el orden social. Así, presumimos que los actores: (a) perciben de manera razonablemente adecuada el funcionamiento del orden, así como (b) conocen también el contenido del consenso normativo y, por lo tanto, (c) evalúan adecuadamente el ajuste entre ambos elementos. El segundo mecanismo social de legitimación, al que llamaremos el mecanismo de disimulación, cuestiona el primer punto.

El mecanismo de disimulación puede rastrearse hasta algunas interpretaciones del concepto marxista de ideología basadas en la idea de falsa consciencia. Con frecuencia, se atribuyó erróneamente a Marx la idea de que las contradicciones del capitalismo inducían en las personas una consciencia errada que les impedía percibir adecuadamente las relaciones sociales reales, y su propia posición en ellas. Así, las personas desarrollarían una falsa consciencia respecto de sus intereses. Esta noción, realmente introducida por Lukács (1923), generó un extenso debate sobre las implicaciones epistemológicas del concepto marxista de ideología (Ricoeur 1986; Mannheim 1936). Sin embargo, la noción de que percibimos de manera distorsionada la realidad y de que dicha distorsión permite la legitimación del statu quo goza de aceptación en tradiciones teóricas bastante diversas.

Notablemente, Bourdieu (2000) resalta la opacidad de la arbitrariedad de las relaciones y distinciones sociales, lo que permite a los privilegiados disimular el origen de su posición mediante el uso de capital simbólico. El capital simbólico consiste en la disimulación de otras formas arbitrarias de capital bajo formas que son afines al consenso normativo, que conllevan merecimiento. Luc Boltanski (2011) y la sociología pragmática francesa critican a Bourdieu por menospreciar la capacidad crítica de los agentes, pero igualmente explicarán el "exceso" de justificación de sistemas arbitrarios mediante la opacidad de las relaciones sociales (Chiapello 2003). La teoría del mundo justo (Lerner 1980) propone que los actores tienen una preferencia por creer que el mundo es justo, por lo que distorsionan su propia percepción para reducir la disonancia generada por situaciones injustas. Similarmente, la teoría de la justificación del sistema (Jost, Banaji y Nosek 2004) propone que la justificación de los sistemas opresivos resulta de aprendizajes sociales que los actores en posiciones desventajadas experimentan como mecanismo paliativo de la disonancia cognitiva que supone comprender su propia posición.

En suma, diversas teorías explican la capacidad de los órdenes sociales de asegurar su validez social mediante la distorsión sistemática de nuestras percepciones acerca de la naturaleza del propio orden. Así, un orden social puede ser incapaz de consolidar un consenso normativo que justifique su funcionamiento concreto, pero puede producir, en cambio, un consenso cognitivo sobre este funcionamiento que sí se ajusta al consenso normativo plausible. De un lado, el consenso normativo y, del otro, las estructuras y relaciones sociales están en conflicto recíproco, pero dicho conflicto queda disimulado por el carácter distorsionado del consenso cognitivo. Con sus diferencias, las perspectivas recién esbozadas y otras pueden describirse mediante esta definición mínima del mecanismo de disimulación. Dicha definición mínima se presenta esquemáticamente en la figura 3 .

En este caso, el consenso normativo se encuentra en conflicto $(\neq)$ con el orden social y sus estructuras: el contenido del primero no permite justificar el funcionamiento del segundo. Por tanto, el grado y forma en que el orden determina el consenso normativo es secundario para el mecanismo de disimulación. 
Figura 3.

Mecanismo de disimulación

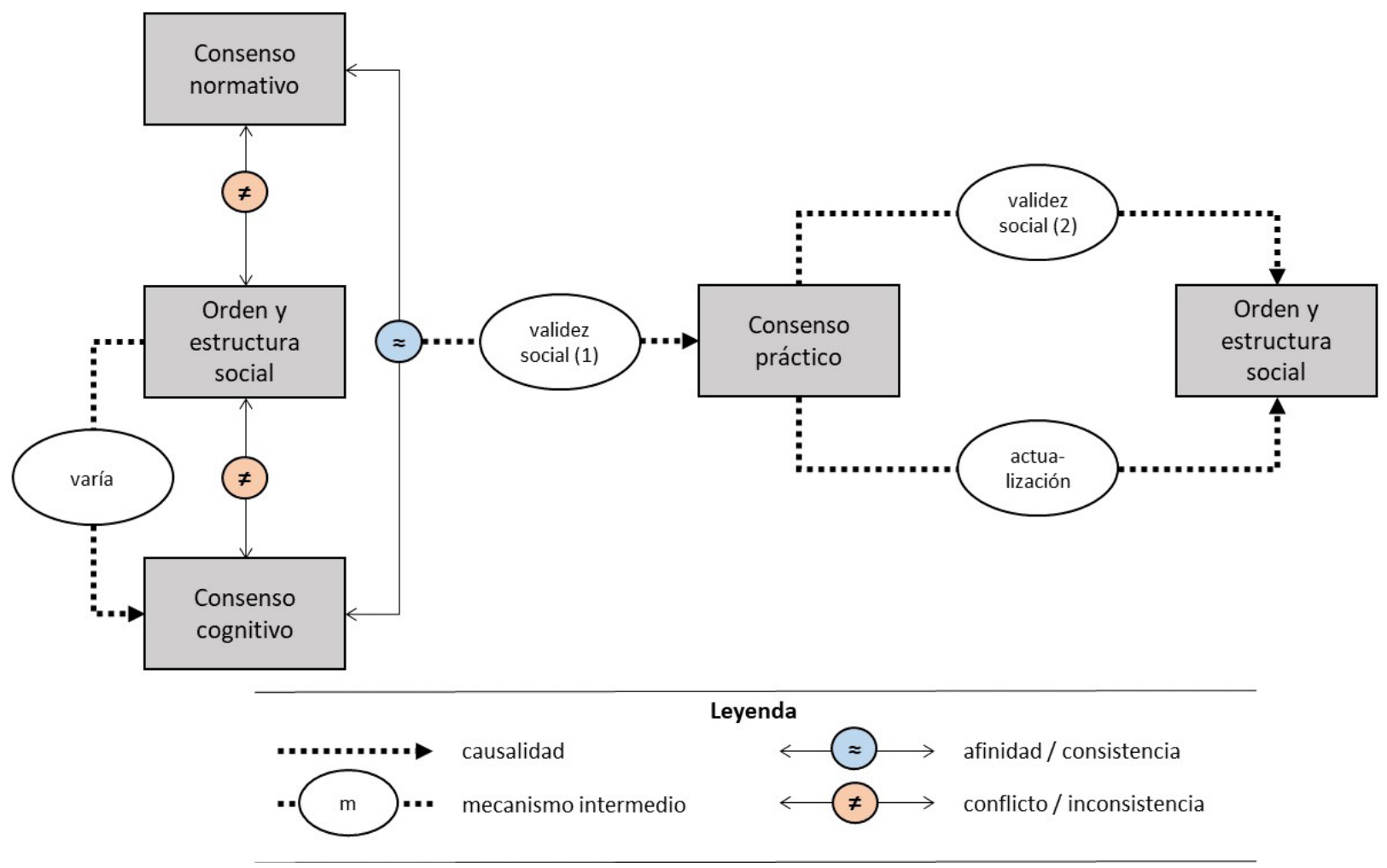

El orden social asegura -mediante mecanismos intermedios que varían de acuerdo a distintas perspectivas teóricas - un consenso cognitivo distorsionado $(\neq)$ respecto del orden social, que sí posee afinidad $(\approx)$ con el consenso normativo. Esta afinidad entre el consenso cognitivo y el normativo permite, apoyándose en el mecanismo de la validez social (1), la reproducción estable del consenso práctico y, por tanto, del orden social mismo ${ }^{2}$. Como sucede con el mecanismo de legitimidad social, este consenso práctico a su vez refuerza permanentemente la validez social (2) del orden, al tiempo que lo reproduce concretamente.

En el ejemplo de la mina, en este caso los trabajadores consideran justo que el Estado y el propietario definan las normas de trabajo, siempre que las ganancias sean distribuidas de forma equitativa entre este último y todos los trabajadores. Consideran injusto que el propietario se enriquezca más allá de esto a partir del trabajo de todos, aunque sea el propietario de la mina. Los trabajadores, sin embargo, ignoran cuántas ganancias retira el propietario. Asumen que el dinero se distribuye de esta manera equitativa, cuando en realidad el propietario ha acumulado mucho más de lo que sería proporcional a su contribución en el proceso de trabajo, dinero con el que invierte en otros lugares. Además, el propietario establece los salarios en el mínimo necesario para mantenerles trabajando. Si los trabajadores en su conjunto se percataran de todo esto, estallaría un conflicto en la mina: sin embargo, como en general los trabajadores no se percatan del problema, conti- núan obedeciendo y trabajando. Si un trabajador individual se convenciera de que el propietario se está enriqueciendo a su costa, o sencillamente decidiera que, de cualquier modo, es injusto que el propietario establezca su salario y las reglas de trabajo, la obediencia generalizada de sus pares seguiría siendo suficiente para disuadirle de rebelarse contra el orden de la mina.

Hemos diferenciado hasta aquí el funcionamiento de dos grandes mecanismos de legitimación que comprenden gran parte de las hipótesis sociológicas respecto al problema. Ambos mecanismos admiten variaciones importantes, en particular respecto de cómo los órdenes sociales producen los consensos normativos y cognitivos involucrados. Nuestro interés no es profundizar ahora en estas variaciones, sino delimitar estos mecanismos generales y mostrar que se puede identificar un tercer mecanismo que, hipotetizamos, tiene mayor potencial explicativo.

\section{LA IDEOLOGÍA COMO INVERSIÓN EN KARL MARX}

Como indicamos inicialmente, Marx no desarrolló sistemáticamente un concepto de ideología ni una teoría sobre cómo el capitalismo aseguraba la cooperación de la clase trabajadora. Nuestro objetivo no es revisar la evolución del concepto de ideología en Marx, sino reflexionar sobre sus posibilidades explicativas y dotarle de una formulación analítica más clara en relación con los procesos de legiti- 
mación. No pretendemos, entonces, capturar toda la complejidad del pensamiento de Marx sobre el tema. En cambio, proponemos una formulación del mecanismo ideológico de legitimación basado en las tesis de Marx sobre lo que podemos llamar 'fenómenos ideológicos'.

Los fenómenos ideológicos remiten a la articulación espontánea entre las relaciones materiales y la formación de las ideas, y a cómo esta articulación permite la reproducción de dichas relaciones materiales. Esta definición genérica ya introduce dos principios básicos: (a) la ideología para Marx no es un concepto neutro, a diferencia de cómo lo desarrollarán otros marxistas posteriores (cfr. Gramsci 1971; Lukács 1923; Lenin 1902), sino que se vincula con la reproducción de la explotación; (b) la ideología no es un fenómeno ideal, sino una articulación entre la vida material y la de las ideas. Articulación espontánea, en cuanto la ideología describe una dinámica que es interna al vínculo entre relaciones sociales capitalistas y la formación de las ideas, que no requiere - como en Weber- de la intencionalidad de actores individuales ni colectivos para producirse, ni implica tampoco la incapacidad de los actores de percibir adecuadamente el orden social -como en Bourdieu-. Cuando estudiamos el problema de la legitimación desde Marx, el locus se desplaza desde el desarrollo de la consciencia de los actores a la relación entre actores y estructuras.

Rastreamos la preocupación de Marx sobre la ideología hasta sus críticas del concepto de Estado en Hegel, y de la religión. En ambos casos Marx afirma que se invertía el funcionamiento de la realidad. Para Hegel, el Estado prusiano solo podía ser la realización material de una idea y, por lo tanto, la idea del Estado solo podía reflejar la abstracción correspondiente a dicho Estado concreto. Aquí, Marx identifica una inversión de la relación entre historia material y consciencia, que silenciosamente hacía imposible advertir un potencial conflicto entre idea y realidad. Similarmente ocurre cuando Marx (1844) reflexiona sobre la religión, en cuanto expresión de y protesta contra el sufrimiento material de las personas: quienes buscaban explicar la religiosidad de las personas mediante sus ideas e ilusiones confundían síntomas con causas.

Con La ideología alemana, Marx y Engels (1846) desarrollan una reflexión más sistemática sobre el punto. Como destaca Althusser (1970), es un texto cuyas analogías ambiguas resultaron en interpretaciones de lo ideológico como cognición fallida o falsa consciencia. Sin embargo, encontramos aquí también dos proposiciones clave sobre el problema de la formación de las ideas: (a) el materialismo explica la formación de las ideas a través de prácticas materiales, en lugar de explicar las prácticas a partir de las ideas; (b) la dominación material lleva a la dominación ideal.
La primera de estas tesis establece que la experiencia material de las relaciones sociales determina los límites y tendencias de la formación de las ideas, y que estas experiencias, en última instancia, están delimitadas por las relaciones sociales de producción. La segunda a menudo se ha confundido con la idea de que las clases dominantes producen e imponen activamente su propia ideología, cuando concretamente lo que Marx afirma es que el control sobre las condiciones materiales de producción conlleva, necesariamente, el control sobre las condiciones en que las clases oprimidas desarrollan sus propias ideas. En efecto, estas tesis no versan sobre la ideología en específico, sino sobre las ideas en general, un hecho que intérpretes como Althusser (1970) o Abercrombie y Turner (1978) han obviado. En cambio, estas tesis describen la perspectiva materialista opuesta al funcionamiento de la ideología: Marx afirma que los ideólogos buscan, encuentran, explican y resuelven contradicciones en la consciencia de los actores sociales, cuando debieran explicar estas contradicciones en su vida material. Es en esto que consiste la ideología: la inversión de la relación entre vida material e ideal.

En El Dieciocho Brumario de Louis Bonaparte, Marx (1852) añade una tercera proposición: sobre la base de las relaciones materiales de producción se levanta una superestructura de sentimientos, expectativas y formas de pensamiento. Marx (1859) especifica después esta propuesta, distinguiendo entre: (a) una estructura social, que corresponde a relaciones sociales en las que entramos con independencia de nuestra voluntad; (b) una superestructura legal y política, y (c) formas de pensamiento que son afines a dicha superestructura. La superestructura no está determinada por las relaciones sociales de producción (todas las relaciones sociales realmente determinadas son, por definición, parte de la estructura), pero es elaborada sobre la base de esta. Althusser (1970) expresaría esto como la autonomía relativa de la superestructura.

Lo central aquí es que, en contraste con diferentes interpretaciones (Abercrombie y Turner, 1978; Althusser, 1970; Plekhanov, 1895), ni la superestructura es una suerte de nivel ilusorio o menos material de la realidad, ni lo ideológico corresponde a lo superestructural o algún nivel de la superestructura. En cambio, proponemos que la ideología es una forma específica en que se articulan estructuras, superestructuras y formas de consciencia. Las relaciones entre los distintos elementos de esta configuración aparecen invertidas (no así los contenidos de las estructuras sociales: no se trata de una distorsión perceptual). En la ideología, por ejemplo, el sistema liberal democrático y el estado capitalista existen porque existen creencias compartidas sobre su justificación, y las relaciones capitalistas de producción existen como resultado del funcionamiento democrático de 
la superestructura institucional porque también la preferimos. En realidad, desde esta perspectiva, estas justificaciones se vuelven hegemónicas porque estamos sujetos materialmente por la existencia de relaciones capitalistas de producción, un estado capitalista y un sistema liberal democrático.

Es esta inversión ideológica la que dota artificialmente de validez social al orden capitalista. En efecto, Marx no llama ni a la justificación del Estado ni a la religión ideologías: en cambio, sí afirma que Hegel era un ideólogo cuando invertía la relación entre el Estado concreto y la idea de Estado, y que los idealistas alemanes hacían ideología cuando invertían la relación entre la religión y las condiciones materiales de existencia. Para Marx, las formas de consciencia de los actores sociales están prefiguradas por las relaciones sociales que experimentan, en buena parte con independencia de su voluntad. Para la ideología, en cambio, las relaciones materiales son resultado de nuestras formas de consciencia y son, por tanto, siempre socialmente válidas y humanamente naturales. Esta es la base del mecanismo de inversión ideológica.

Para Marx, entonces, la ideología no es nunca un conjunto de ideas o disposiciones que nos llaman a actuar de determinada forma (Eagleton 1991) - y he aquí una diferencia fundamental con el mecanismo de legitimidad social—. La ideología, en cambio, es un fenómeno que impide el despliegue de ciertas prácticas concretas: fenómeno que no pertenece completamente al mundo de las ideas.

El Marx temprano reflexiona sobre la ideología principalmente refiriéndose a teorías específicas. Aunque la ideología represente una articulación de distintos niveles de la realidad, en su estudio concreto realiza únicamente elaboraciones teóricas. Esta tensión no será abordada explícitamente por Marx, que irá abandonando el concepto. Sin embargo, la idea de inversión y su capacidad de estabilizar el funcionamiento del capitalismo sigue presente en sus análisis posteriores. En efecto, podemos elaborar una formulación más clara de la ideología como mecanismo de legitimación cuando atendemos a su estudio posterior de la acumulación del capital.

Aunque no encontremos la palabra ideología en el análisis marxiano de la acumulación capitalista, diversos autores (Larraín 2007; Eagleton 1991; Žižek 1989) identifican aquí un núcleo teórico clave para entender sus ideas al respecto: núcleo que ya no está circunscrito a la crítica de las teorías ideológicas, sino que proporciona un método para analizar las formas cotidianas de la práctica ideológica. Este núcleo está en la idea de fetichismo. En particular, el trabajo de Žižek (1989) debe reconocerse por la clarificación de la noción de inversión fetichista y su relación con el mecanismo ideológico en general. No obstante, desde su matriz psicoanalítica, termina por psicologizar y desdibujar el problema.
El análisis del fetichismo de la mercancía en El Capital describe una inversión ideal que se explica por una inversión material: la alienación. Mientras para Hegel (1807) la alienación era una externalización necesaria en el proceso de objetivación, para Marx es una forma específica de objetivación en que el trabajo objetivado se vuelve autónomo y hostil respecto de sus productores. La alienación, entonces, es una inversión material, específica del capitalismo y que posee implicancias perversas. Esta inversión material lleva al fetichismo (inversión ideal) cuando la relación de los productores con su propio trabajo se les presenta como una relación entre las mismas mercancías. Sin embargo, Marx reconoce que este no es un descubrimiento nuevo, ya que la economía política había analizado la naturaleza del valor detrás de la forma mercancía: lo que los economistas no habían hecho, en cambio, era preguntarse por qué el trabajo se presentaba ante los productores de esta forma (Marx 1894: 86-87).

El problema ideológico, entonces, no es lo que está escondido detrás de la forma (i.e., la ideología como si fuera falsa), sino la forma misma y por qué se desarrolla (Žižek 1989). La economía política aceptaba esta forma como natural, cuando históricamente era evidentemente específica; observar esta evidencia, sin embargo, nada avanza en desmontar la inversión fetichista, puesto que está anclada en una inversión material. La apariencia de naturalidad de la mercancía no depende de la consciencia de los productores, sino que está anclada en las relaciones de producción en las que estos participan con relativa independencia de su voluntad. En efecto, los trabajadores no necesitan creer que su trabajo no produce valor, ni que el valor se produce en el momento del intercambio, basta con que realicen esta inversión en su práctica concreta: el efecto ideológico no se produce porque creamos en él, sino porque lo practicamos y, dadas ciertas estructuras, no podemos hacerlo de otra forma.

A través del fetichismo, el valor abstracto cobra existencia por fuera de las relaciones de intercambio y la mercancía adquiere propiedades que son humanas. El despliegue de prácticas le da poder y realidad a esta inversión, que los individuos no son libres de ignorar o negar a voluntad. Al mismo tiempo, y esto es clave para el mecanismo de inversión, nuestra práctica concreta se convierte en la prueba permanente de la verdad de la inversión ideológica. Así, tomando la voz de las mercancías, Marx afirma en El Capital: "lo que sin embargo nos pertenece en tanto objetos es nuestro valor: nuestro intercambio natural como mercancías lo prueba" (Marx 1894: 95).

Entendiendo el análisis del fetichismo como una aplicación del problema de la ideología, entonces, tenemos lo siguiente. En una sociedad capitalista existen una serie de inversiones reales, i.e., relaciones sociales que encierran contradicciones internas. Los 
individuos están compelidos a participar en estas relaciones actualizándolas en su práctica, con relativa independencia de su voluntad. Siendo contradictorias, estas relaciones sociales empujan a las personas a desarrollar una consciencia crítica de ellas y, al mismo tiempo, a desarrollar explicaciones respecto de su reproducción. A partir de estas prácticas materialmente predeterminadas, entonces, las contradicciones materiales del orden aparecen como el resultado de contradicciones ideales en la consciencia de los sujetos. Actuamos de manera aparentemente contradictoria con nuestros propios intereses porque estamos anclados a relaciones materiales en sí contradictorias, pero la ideología presenta esta relación de manera inversa: como si nuestra realidad material fuera contradictoria porque actuamos de acuerdo a una consciencia contradictoria.

\section{FORMULANDO EL MECANISMO DE IN- VERSIÓN IDEOLÓGICA}

¿Cómo traducimos esta noción marxiana de ideología en un esquema similar al de los otros mecanismos de legitimación? En el mecanismo de legitimidad social, el consenso normativo explica el alineamiento de las personas con el orden - expresada en un consenso práctico- a través del mecanismo intermedio de la validez social. Sin embargo, el consenso normativo es una abstracción difícil de observar directamente: los actores solo pueden inferir su existencia a partir de su materialización en el consenso práctico. Este proceso de inferencia es, en realidad, una inversión ideológica. Incluso si los actores reproducen en su práctica relaciones que están en conflicto con sus formas de pensamiento, que no se ajustan ni son justificadas por su valores y creencias, el mecanismo de validez social se gatilla. Realizamos la ideología en la práctica y, parafraseando a Žižek (1997), creemos en la validez del orden a través de las prácticas de los otros.

No se requiere, así, de una falsa consciencia de la realidad para dar cuenta de la ideología. Los actores pueden ser perfectamente conscientes de que están inmersos en relaciones de opresión y explotación de diverso tipo y, aun así, operar bajo la lógica de la inversión ideológica. En efecto, los individuos incluso pueden estar conscientes de la inversión ideológica misma, comprendiendo que el orden social no goza de una verdadera validez social. Esto en nada socava la validez social que el orden adquiere en la práctica: actuar individualmente en contra del orden seguirá teniendo todas las consecuencias negativas y los escasos efectos transformadores de actuar contra un orden legítimo. La inversión ideológica explica la legitimación a través del poder sordo de las relaciones sociales sobre nuestra conducta y de la capacidad de dicha conducta de comunicar acuerdo con independencia de si dicho acuerdo existe realmente.
Los tres mecanismos de legitimación implican que las prácticas de los individuos reproducen el orden aunque no tengan motivaciones individuales para hacerlo. Estos individuos se alinean con el orden mediante el mecanismo de la validez social, que es el que precisamente dota de estabilidad a cualquier proceso de legitimación. En el caso de la inversión ideológica, sin embargo, este caso es el generalizado, e incluso quienes actúan de acuerdo genuino con el consenso normativo socialmente percibido, en realidad actúan de acuerdo con un consenso fantástico que solo existe como expresión ideal del consenso práctico definido por las estructuras sociales.

Así, la fractura entre sus propias prácticas y su consciencia solo es observable para el individuo mismo. En la experiencia social, cada uno parece actuar de acuerdo a una consciencia compartida de lo que es real y justo: experimentamos la práctica de los otros como si fuera consistente, pues no tenemos acceso permanente y directo a sus propios conflictos. Así es como emerge el consenso fantástico y reemplaza un consenso normativo que, de existir, se torna invisible en tanto no se realiza prácticamente.

Como hicimos con los otros mecanismos de legitimación, presentamos esquemáticamente el mecanismo de inversión ideológica en la figura 4. En primer lugar, existe una relación de conflicto e inconsistencia $(\neq)$ entre el orden social y el consenso normativo, pues las sociedades capitalistas presionan a los actores a desarrollar valores y creencias en conflicto con el contenido del orden: el capitalismo es incapaz de producir un acuerdo normativo que lo justifique adecuadamente. En cambio, existe una relación de afinidad $(\approx)$ entre el orden social y el consenso cognitivo: esto señala que, para la operación del mecanismo de inversión ideológica, no es requisito que los actores desarrollen una percepción distorsionada del orden. Esto puede ocurrir, pero la legitimación ideológica del orden no depende de cuán distorsionada sea la percepción que estos tengan de la realidad material.

Por lo mismo, existe una relación conflictiva $(\neq)$ entre el consenso cognitivo y el normativo: la percepción que las personas comparten sobre la realidad no coincide con los valores y preferencias que comparten. De expresarse el conflicto entre el consenso normativo y el consenso cognitivo, la reproducción del consenso práctico debiera interrumpirse: no existe un primer momento de validez social (1).

El consenso práctico, sin embargo, no se interumpe, ya que partes claves de este vienen determinadas directamente por la misma estructura social. Esta determinación es imperfecta, pero suficiente para que el consenso normativo se haga invisible ante los mismos actores que lo comparten y, en cambio - a partir de la inversión ideológica-, aparezca ante ellos un consenso fantástico que es consistente $(\approx)$ con el orden. El consenso fantástico no es más 
Figura 4.

Mecanismo de inversión ideológica

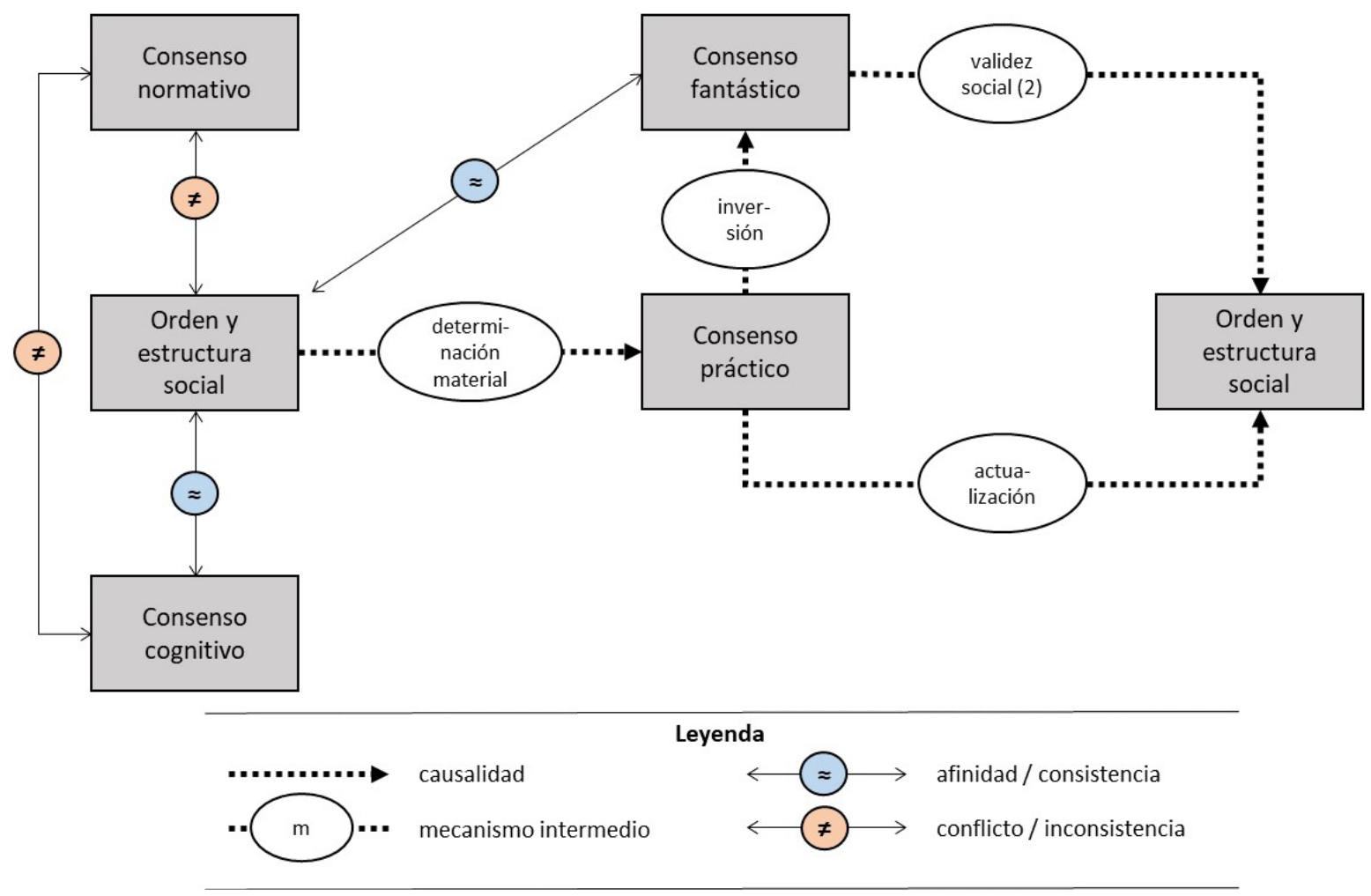

que la mistificación del consenso práctico (cfr. Burawoy 2012), reforzándolo y permitiéndole gatillar el mecanismo de validez social (2).

Para simplificar el esquema hemos ubicado el consenso fantástico al lado del consenso normativo, reemplazándolo e invisibilizándolo. Sin embargo, el consenso fantástico es igualmente capaz de reemplazar e invisibilizar el consenso cognitivo. Cuando obedecemos ideológicamente a partir del consenso de los otros, podemos hacerlo porque atribuimos a estos otros valores que justifican el statu quo - como si existiera legitimidad social- o percepciones sesgadas que justifican el statu quo -como si existiera disimulación-. Para efectos de la capacidad estabilizadora de la inversión ideológica, la diferencia es menor $y$, probablemente, esta opera siempre mediante una combinación de ambos efectos.

Volviendo al ejemplo de la mina, en el caso de la inversión ideológica la gran mayoría de los trabajadores posee individualmente valores igualitaristas $y$ considera sumamente injusto que el propietario defina las reglas del trabajo, así como que se apropie de las ganancias de la mina. Individualmente, también son conscientes de que el propietario efectivamente retira ganancias y se lucra así con el resultado de su trabajo. Sin embargo, requieren urgentemente del salario que ofrece el propietario para subsistir y, por lo tanto, cada uno en su momento ha aceptado esta situación. Ninguno de ellos aceptaría este trato si tuviera completa libertad para hacerlo, pero ninguno ha estado nunca en esa posición. El problema es que estos mismos trabajadores observan la obediencia y el silencio de sus colegas como expresión de su acuerdo con las normas de la mina o, tal vez, como expresión de que estos colegas están completamente engañados por el patrón. A partir de esta inferencia, de esta inversión, actúan de forma conformista bajo la idea de que el orden social de la mina está socialmente validado por sus pares.

El mecanismo de inversión ideológica es una estrategia explicativa marcadamente diferente a las que hallamos en otras tradiciones sociológicas y a las desarrolladas desde la psicología social. La exposición analítica de los tres mecanismos busca clarificar el funcionamiento de la ideología con el fin de que su potencial explicativo pueda evaluarse empíricamente y compararse con el de otras teorías sobre la legitimación.

Un estudio sistemático de los mecanismos de legitimación requiere, desde esta perspectiva, atender no solo a las ideas de los actores sino, sobre todo, a la relación de: (a) las prácticas propias y las prácticas de los otros con (b) mis propias ideas y las ideas que atribuyo al resto de la sociedad. Implica, así, un retorno al carácter social e interaccional del proceso de legitimación en el estudio de la legitimación del orden.

Esta agenda de investigación debe abordar, al menos, cuatro procesos: (a) cómo las estructuras e instituciones moldean interacciones y prácticas que comunican su propia legitimidad, en concordancia con o pese a las preferencias de los acto- 
res; (b) cómo las prácticas moldean las preferencias atribuidas a terceros y al colectivo; (c) cómo las posiciones sociales (de clase, género, etc.) moderan la configuración de preferencias atribuidas y propias y, finalmente, (d) el impacto de la atribución de ideas legitimantes a los otros en la probabilidad de cuestionar el statu quo mediante prácticas concretas (individuales o, sobre todo, colectivas). Buscamos explicar prácticas, y no preferencias o actitudes, ya que las prácticas reinician el ciclo de la legitimación. Debemos reconstruir el proceso que reactiva permanentemente la producción del consenso práctico, rastreando las consistencias e inconsistencias entre el consenso normativo, el consenso cognitivo y, (de demostrarse su existencia), el consenso fantástico.

En general, la literatura internacional omite el rol de las preferencias atribuidas a los otros en la legitimación del capitalismo (proceso $d$ ) y cómo las posiciones sociales de los actores moderan esta atribución (proceso $c$ ). El proceso $b$ apenas ha recibido atención, con la brillante excepción de los estudios de Burawoy (1979) en el contexto de la fábrica. El proceso a es el único estudiado más sistemáticamente, especialmente desde la teoría de la elección racional (Klandermans 1984; Olson 1971) y el marxismo analítico (Elster 1985; Roemer 1982), así como por Bourdieu en el ámbito del sistema educativo (Bourdieu y Saint Martin 1998; Bourdieu y Passeron 1990).

\section{LAS HIPÓTESIS DE LA INVERSIÓN IDEOLÓGICA}

Tras proponer esta agenda investigativa para unificar analíticamente el estudio de la legitimación de las sociedades capitalistas, exponemos las principales hipótesis concretas que se desprenden del mecanismo de inversión ideológica.

$\mathrm{H} 1$ : Los individuos tienden a atribuir a terceros ideas (preferencias o percepciones) sistemáticamente más orientadas a la reproducción del orden social que las que estos terceros efectivamente poseen.

Esto porque las personas infieren, a partir de la adaptación a las estructuras sociales que realizan otros, ideas consistentes con dichas conductas. Las personas están constreñidas a adoptar prácticas que les permiten sobrevivir y mejorar sus condiciones en ciertas estructuras ya dadas, y estas mismas prácticas son interpretadas como expresión de una preferencia por el statu quo. Si bien existen, en toda sociedad, conductas desviadas que cuestionan la validez social del orden, una estructura social estable tenderá a reducir estas expresiones y contrarestarlas adecuadamente.

En el agregado social, esto implica la diferenciación entre un consenso normativo (las ideas compartidas por el colectivo) y un consenso fantástico (las ideas que los individuos infieren que el colectivo comparte). Por ejemplo, en una sociedad caracterizada por un modelo económico de libre mercado, se tenderá a atribuir a otros ideas más libremercadistas que las que poseen. Para sobrevivir y adaptarse en un contexto de libre mercado, las personas actúan de acuerdo a principios mercantiles de conducta: de esto se deriva la apariencia de que el libre mercado existe debido a a las orientaciones de la conducta de las personas.

H2: Parte de la distorsión en la atribución de ideas a terceros se explica por la adopción de prácticas que están mayormente explicadas por la adaptación (racional) a las estructuras sociales.

Esta segunda hipótesis puede ejemplificarse con un contexto capitalista en que confluyan la desregulación laboral y un modelo cultural patriarcal. Si las mujeres reciben sistemáticamente menos recompensas por tomar un empleo remunerado que sus pares hombres, para amplios sectores sociales la no participación de las mujeres en el mercado laboral más que una opción es el resultado esperable de un cálculo de altos costos (trabajo doméstico, cuidados) versus escasos beneficios (salarios inequitativos). De todas formas, las personas tenderán a atribuir ideas más conservadoras a las mujeres que no participan del mercado laboral y a sus cónyuges, a partir de la observación de la práctica de trabajos domésticos no remunerados. Dado que el diferencial de costos y beneficios es peor cuanto peor remunerado está el trabajo, esto redunda en que se atribuyen finalmente ideas más conservadoras a las clases bajas.

Una hipótesis secundaria $(\mathrm{H} 2.1)$ que se deriva de esto es que en una sociedad, cuanto más penetra la lógica del mercado en distintas esferas de la vida (educación, cuidados, pensiones, salud, sistema de partidos, el mismo trabajo, etc.), al verse más compelidas las personas a actuar racionalmente bajo dichos arreglos, mayor será la distancia entre el consenso normativo y el consenso fantástico. Siguiendo con el último ejemplo, si en una sociedad existe un sistema universal de cuidados que no pasa por la inversión familiar de recursos, menos mujeres se excluirán del mercado laboral y la sociedad misma se percibirá colectivamente como menos conservadora en este aspecto.

H3: La atribución de ideas a los otros, sistemáticamente distorsionada hacia ideas afines al statu quo, tiene importantes efectos inhibidores de las prácticas que son disruptivas del orden social. Es decir, mientras una persona más tiende a atribuir ideas excesivamente legitimantes a los demás, menos participará en acciones disruptivas del orden. Esto, aún después de controlado el efecto de sus propias ideas y otros determinantes conocidos de la acción por el cambio social. 
Esto debiera observarse en prácticas y acciones individuales como el voto en las elecciones, pero sobre todo en la participación en acciones colectivas disruptivas. En un nivel agregado, esto implica que a mayor distancia entre el consenso normativo y el consenso fantástico, menos expresiones de conflictividad se observan a partir de los conflictos entre las estructuras sociales y las preferencias e intereses de las personas. Así, por ejemplo, cuanto más racistas se perciban entre sí los individuos en una sociedad, menos expresiones de protesta y acción política contra las desigualdades raciales observaremos en ella, una vez considerado el efecto de cuán fuertes son las disposiciones racistas efectivamente en la población.

A esta hipótesis final podemos asociar una última hipótesis secundaria (H3.1): la relevancia ideológica de las grandes acciones colectivas. Mientras el consenso fantástico inhibe las acciones colectivas contra el statu quo, cuando se producen con suficiente frecuencia y escala estas acciones debieran incidir en la forma en que los individuos perciben a sus pares y sus ideas. Esto implica que, en una sociedad que recientemente ha experimentado oleadas fuertes de acción colectiva, la distancia entre el consenso normativo y el consenso fantástico debiera reducirse, abriéndose una ventana de oportunidad para el resquebrejamiento de la legitimación ideológica del orden. De comprobarse esta hipótesis, se podría establecer un vínculo más estrecho entre las dinámicas de la acción contenciosa y los estudios de la legitimación del orden social.

Si bien ya es posible elaborar análisis preliminares promisorios sobre estas hipótesis (Puga, en prensa), producir evidencia sistemática para ellas requiere instrumentos específicos, diferentes a los comúnmente utilizados en la investigación sobre la legitimación de la desigualdad social (en general, estos instrumentos recaban información únicamente sobre disposiciones individuales, omitiendo la atribución de ideas a terceros). De producirse, esta evidencia podría tener un impacto considerable en cómo entendemos la reproducción del capitalismo y las acciones orientadas a su transformación.

\section{IMPLICACIONES FINALES}

Por una parte, el mecanismo de inversión ideológica obliga a retornar a los aspectos sociales e interaccionales que los clásicos de la teoría social consideraban centrales para la reproducción del orden. Por otra, ofrece una alternativa sólida al extendido conflicto entre una sociología crítica que subestima la capacidad crítica de los agentes y una sociología de la crítica (Boltanski 2011) que subestima el poder coercitivo de las estructuras sociales sobre estos.
Este mecanismo permite también abordar regularidades que resultan contraintuitivas desde las perspectivas de la legitimidad social o de la disimulación, principalmente, la sorprendente estabilidad (en perspectiva comparada) de sociedades particularmente desiguales y segregadas (Bucca 2016).

Colocando el foco en la diferenciación entre preferencias compartidas y preferencias percibidas como compartidas, el mecanismo de inversión ideológica también permite desarrollar y complejizar la relación entre los estudios de la legitimación del orden y la desigualdad, de un lado, y del otro los estudios de la acción colectiva; esta última, potencialmente relevante para la modificación de las preferencias percibidas como compartidas.

Finalmente, resulta pertinente discutir el papel del mecanismo dentro de una teoría marxista del cambio social. Este mecanismo no pretende reemplazar la discusión sobre los conflictos de clases y la capacidad de estas para conducir o desbaratar procesos de transformación social (cfr. Heller, 2017). En cambio, elabora una teoría sociológica explícita a partir de Marx (1867) sobre cómo las estructuras sociales tienden espontáneamente a estabilizarse a partir de su "fuerza sorda".

El mecanismo ideológico explica la dificultad inherente para que se inicien procesos de movilización que pueden resultar en transformaciones sociales, dificultad que no requiere de las clases dominantes un trabajo ni una coordinación activa. La cohesión y coordinación de las clases enfrentadas en dichos procesos, por otra parte, incidirán en el desarrollo de estos. El funcionamiento del mecanismo ideológico permite entender también por qué, aun si estas movilizaciones no producen directamente transformaciones sociales, pueden resquebrajar la legitimación del orden y favorecer el surgimiento de nuevas movilizaciones en el futuro. Así, nuestra formulación busca tanto complementar como complejizar la comprensión de los procesos de estabilidad y transformación social.

\section{Agradecimientos}

Este trabajo se ha realizado con el apoyo de la Comisión Nacional de Ciencia y Tecnología (CONICYT) de Chile, en el marco de los Proyectos Fondecyt "Prácticas sociales y mecanismos de legitimación de la desigualdad en Chile", CONICYT/FONDECYT/11160413, y "Justicia, legitimidad y cambio social en el ámbito laboral”, CONICYT/FONDECYT/ 1171072. Este trabajo contó también con apoyo del Centro de Estudios de Conflicto y Cohesión Social, CONICYT/FONDAP/15130009, COES. 


\section{NotAs}

[1] Sin embargo, introduce aún considerable ruido si se quiere estudiar la legitimación de un orden, dado que las sensaciones de injusticia poseen numerosas explicaciones contingentes más allá de la falta de justificación de dicho orden en general.

\section{REFERENCIAS BiBLIOGRÁFICAS}

Abercrombie, N. y B. Turner. 1978. "The Dominant Ideology Thesis". The British Journal of Sociology 29(2): 149-170.

Almstrom, C. 2006. "A Three-Worldview of System Justification Theory". Journal of Scientific Psychology (September): 6-8.

Althusser, L. 1970. "Ideology and Ideological State Apparatuses". Pp. 127-187 en Lenin and Philosophy, and other essays, editado por Althusser, L. New York: Monthly Review Press.

Arnett, R. y J. Sidanius. 2018. "Sacrificing status for social harmony". Organizational Behavior and Human Decision Processes 147: 108-126.

Beetham, D. 2013. The Legitimation of Power. London: Macmillan.

Bénabou, R. y J. Tirole. 2006. "Incentives and Prosocial Behavior". The American Economic Review 96(5): 16521678.

Berger, J. y M. Zelditch. 1998. Status, power, and legitimacy. New Brunswick: Transaction.

Boltanski, L. 2011. On critique: A sociology of emancipation. Cambridge: Polity

Boltanski, L. y E. Chiapello. 1999. The new spirit of capitalism. London: Verso.

Boltanski, L. y L. Thévenot. 1999. "The Sociology of Critical Capacity". European Journal of Social Theory 2(3): 359-377.

Bourdieu, P. 1979. "Symbolic Power". Critique of Anthropology 4(13-14): 77-85. https://doi. org/10.1177/0308275X7900401307

Bourdieu, P. 1980. "The production of belief". Media, Culture \& Society 2(3): 261-293. https://doi. org/10.1177/016344378000200305

Bourdieu, P. 2000. Pascalian meditations. Stanford: Stanford University Press.

Bourdieu, P. y J.C. Passeron. 1990. Reproduction in Education, Society and Culture. London: Sage Publications.

Bourdieu, P. y M. Saint Martin. 1998. The state nobility. Cambridge: Polity Press.

Bucca, M. 2016. "Merit and blame in unequal societies". Research in Social Stratification and Mobility 44: 98-112.

Burawoy, M. 1979. Manufacturing consent. Chicago: University of Chicago Press.

Burawoy, M. 2012. "The Roots of Domination". Sociology 46(2): 187-206.

Chiapello, E. 2003. "Reconciling the Two Principal Meanings of the Notion of Ideology". European Journal of Social Theory 6(2): 155-171.

Cichocka, A. y J. Jost. 2014. "Stripped of illusions? Exploring system justification processes in capitalist and postCommunist societies". International Journal of Psychology 49(1): 6-29.
[2] Un consenso cognitivo que describa de forma precisa un orden es improbable: la existencia de "grados" de distorsión en la percepción del orden no indica la primacía explicativa del mecanismo de disimulación. Este mecanismo implica que la contradicción entre el consenso cognitivo y el funcionamiento objetivo del orden social es tal que, de corregirse, la reproducción del orden se interrumpiría.

Durkheim, É. 1893/1964. The division of labour in society. New York: Free Press.

Eagleton, T. 1991. Ideology: An introduction. London: Verso.

Elster, J. 1985. Making sense of Marx. NY: Cambridge University Press.

Foucault, M. 1975/1995. Discipline and Punishment. New York: Vintage.

Gramsci, A. 1971. Selections from the Prison Notebooks. NY: International Publishers.

Hegel, G. 1807/1967. The Phenomenology of Mind. New York: Harper and Row.

Heller, H. (Ed.). 2017. The French Revolution and historical materialism. Boston: Brill.

Homans, G. 1958. "Social Behavior as Exchange". American Journal of Sociology 63(6) 597-606. https://doi. org/10.1086/222355.

Jasso, G. 1978. "On the Justice of Earnings". The American Journal of Sociology 83(6): 1398-1419.

Jasso, G. 2008. "A New Unified Theory of Sociobehavioural Forces". European Sociological Review 24(4): 411434.

Jasso, G. 2015. "The Social Psychology of Immigration and Inequality". Pp. 575-605 en Handbook of the social psychology of inequality, editado por J. McLeod, E. Lawler y M. Schwalbe. Dordrecht, Heidelberg, New York, London: Springer.

Johnson, C., Dowd, T. y C. Ridgeway. 2006. "Legitimacy as a Social Process". Annual Review of Sociology 32(1): 53-78.

Jost, J., Banaji, M. y B. Nosek. 2004. "A Decade of System Justification Theory". Political Psychology 25(6): 881 919.

Klandermans, B. 1984. "Mobilization and Participation". American Sociological Review 49(5): 583-600. https://doi. org/10.2307/2095417

Larraín, J. 2007. El concepto de ideología: Vol. I. Santiago de Chile: LOM.

Lenin, V. 1902/1977. "What is to be done?". Pp. 347-520 en Lenin's Collected Works (Vol. 5). Moscow: Progress Publishers.

Lerner, M.J. 1980. The belief in a just world: A fundamental delusion. New York: Plenum Press.

Lukács, G. 1923/1971. History and class consciousness. Cambridge MA: MIT Press.

Mannheim, K. 1936/1985. Ideology and utopia. San Diego: Harcourt Brace Jovanovich.

Marx, K. 1844/1977. Critique of Hegel's 'Philosophy of Right'. Cambridge: Cambridge University Press.

Marx, K. 1852/1988. The eighteenth Brumaire of Louis Bonaparte. New York: International Publishers. 
Marx, K. 1859/1978. "Preface to the Contribution to the Critique of Political Economy". Pp. 3-6 en The Marx-Engels reader, editado por R. Tucker. New York: Norton.

Marx, K. 1867/1909. Capital: Vol. I. Chicago: Kerr \& Co.

Marx, K. 1894/1909. Capital: Vol. III. Chicago: Kerr \& Co.

Marx, K. y F. Engels. 1846/1998. "The German ideology". En The German Ideology including Theses on Feuerbach and Introduction to The critique of political economy. Amherst NY: Prometheus Books.

Mouffe, C. 1979/2014. "Hegemony and ideology in Gramsci". Pp. 168-204 en Gramsci and Marxist theory, editado por C. Mouffe. London, New York: Routledge.

Müller, H. y B Wegener (Eds.). 1995. Soziale Ungleichheit und soziale Gerechtigkeit. Opladen: Leske \& Budrich.

Olson, Mancur 1971. The Logic of Collective Action. Cambridge, Mass.: Harvard University Press.

Osborne, D. y C. Sibley. 2015. "Opposing Paths to Ideology". Social Justice Research 28(1): 27-51.

Parsons, T. 1979. The social system. London: Routledge \& Kegan Paul.

Plekhanov, G. 1895/1972. The Development of the Monist view of History. New York: International Publishers.

Puga, I. en prensa. "Ideological inversion and the (de)legitimation of neoliberalism in Chile". En The Oxford Handbook of the Sociology of Latin America, editado por L. Rivera y Y. Xochitl Bada. Nueva York: Oxford University Press.
Ricoeur, P. 1986. Lectures on ideology and utopia. New York: Columbia University Press.

Roemer, J. 1982. A general theory of exploitation and class. Cambridge, Mass: Harvard University Press. https:// doi.org/10.4159/harvard.9780674435865

Schneider, S. y P. Valet. 2017. "Relative Standards and Distributive Justice". Social Psychology Quarterly 80(3): 276-287.

Sidanius, J. y F. Pratto. 2001. Social dominance. Cambridge: Cambridge University Press.

Van Zomeren, M., T. Postmes y R. Spears. 2008. "Toward an integrative social identity model of collective action". Psychological Bulletin 134(4): 504-535.

Weber, M. 1918/1999. "The Three Types of Legitimate Domination". Pp. 99-108 en Essays in economic sociology, editado por R. Swedberg. Princeton: Princeton University Press.

Weber, M. 1920/1998. "Domination and Legitimacy”. Pp. 24-37 en German sociology, editado por U. Gerhardt. New York: The Continuum Publishing Company.

Weber, M. 1922/1978. Economy and society: An outline of interpretive sociology. Berkeley: University of California Press.

Wegener, B. 1987. "The Illusion of Distributive Justice". European Sociological Review 3(1): 1-13.

Žižek, S. 1989. The sublime object of ideology. London, New York: Verso.

Žižek, S. 1997. "The Supposed Subjects of Ideology". Critical Quarterly 39(2): 39-59.

ISMAEL PUGA es profesor asociado de la Facultad de Educación y Ciencias Sociales de la Universidad Central de Chile. Licenciado en sociología por la Universidad de Chile y doctor en Sociología por la Humboldt Universität zu Berlin. Sus investigaciones se concentran en el problema de la legitimación del orden y la movilización social en Chile.

CRISTÓBAL MOYA es research fellow de Zeppelin Universität en la cátedra de sociología con foco en el análisis de la estructura social. Es sociólogo, licenciado en Lengua y Literatura Hispánica y magíster en Ciencias Sociales, todos por la Universidad de Chile. Desarrolla actualmente su tesis doctoral sobre procesos de legitimación de la dominación a la luz de la justicia social empírica. 